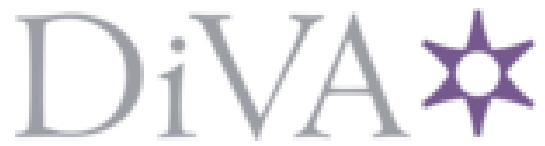

http://www.diva-portal.org

Preprint

This is the submitted version of a paper presented at OCEANS 2019 - Marseille.

Citation for the original published paper:

Torroba, I., Bore, N., Wåhlin, A., Folkesson, J. (2019)

Loop Closure Detection Through Environmental Indicators In Underwater SLAM

In: Marseille France: IEEE

https://doi.org/10.1109/OCEANSE.2019.8867097

N.B. When citing this work, cite the original published paper.

Permanent link to this version:

http://urn.kb.se/resolve?urn=urn:nbn:se:kth:diva-283557 


\title{
Loop Closure Detection Through Environmental Indicators In Underwater SLAM
}

\author{
Ignacio Torroba $^{1}$, Nils Bore ${ }^{1}$, Anna Wåhlin ${ }^{2}$ and John Folkesson ${ }^{1}$
}

\begin{abstract}
A key step in Simultaneous Localization and Mapping (SLAM) with autonomous robots is the ability to recognize when the vehicle is revisiting an area. The recognition of salient features allows re-localization to a previously visited place, forming a so-called loop closure. Loop closures enable autonomous underwater vehicles (AUVs) to reduce the unbounded navigation drift experienced when mapping unknown terrain. However, the scarcity of recognizable features in unstructured sub-sea environments makes disambiguation of places a challenging problem. In this work we study the application of water analysis sensors mounted on an AUV to construct descriptors of underwater environments. Since these sensors measure very different properties of the areas compared to terrain sensors, these descriptors could increase robustness against perceptual aliasing on place recognition and thus improve loop closure detection in a SLAM framework. We present a method to create and compare these spatial descriptors, test it with several data sets collected with an AUV in two different geographical locations and analyze their potential use for loop closure detection.
\end{abstract}

\section{INTRODUCTION AND MOTIVATION}

Autonomous underwater and surface vehicles such as AUVs, drifters and gliders are becoming standard tools for ocean exploration and monitoring of currents, water quality or patterns in species behaviors [1], [2]. Oceanographers and biologists use these vehicles as platforms to collect biochemical data, whose value depends upon being accurately geo-referenced. However, these vehicles suffer drift in the dead-reckoning estimates of motion over time, which GPS can only correct while on the surface. Simultaneous localization and mapping, SLAM, offers a method to reduce that drift even while underwater and exploring unknown environments. Drift around a loop formed when returning to a previously visited place can be reduced if there is some way of recognizing the place. This is a challenging problem as the appearance of the seafloor using sensors such as sonar or cameras is often not enough to discriminate places, leading to false positives. We propose that payload biochemical information, which is being collected anyway, can be utilized to help the place recognition. We would argue that the false positives should not be strongly correlated across sensor modalities so that this will reduce the rate of false positives. Furthermore, in cases where a minimal sensor suite is desirable, one could reduce the number or quality of sensors, following the philosophy introduced in [3].

\footnotetext{
${ }^{1}$ Torroba, Bore and Folkesson are with the Robotics, Perception and Learning Division at KTH Stockholm, Sweden \{torroba, nbore, johnf\}@kth.se

${ }^{2}$ Wåhlin is with the Department of Marine Sciences, University of Gothenburg, Gothenburg, Sweden. anna.wahlin@marine.gu. se
}

State of the art approaches to underwater SLAM exploit the geometry of the sea floor to derive spatial descriptors that can be used to recognize a previously mapped location. These approaches, which build upon sonar or vision technology [4], [5], create models of the environment against which the loop closure search is carried out. They present two main challenges: i) sonar models of unstructured environments are prone to present perceptual aliasing, which causes different places to look the same; ii) the uncertainty in the loop closure search increases with the range of the mission and the size of the loops performed. Generally, to address these challenges, other measurements such as the estimate of the vehicle pose from dead reckoning (DR) are often incorporated to increase the confidence on the loop closure detections [6]. However, these DR estimates can not always be relied upon as they can suffer from unmodeled errors that then lead to poor decisions on loop closures. Thus, a reduction on the dependence on the DR pose estimate is an advantage.

In this paper, to reduce this dependence, narrow down the loop closure search space and increase robustness to perceptual aliasing, we turn to the water analysis sensors carried as part of the scientific payload. We aim to find out if they contain information that can be utilized to construct richer descriptors of the subsea environment. Some of the biochemical indicators being measured are up-welled from benthic or hydrographic processes taking place on the sea floor [7]. Since some of these processes are stationary, we expect to observe time-consistent multimodal distributions of the indicators such that combined together they could help disambiguate areas of sea floor that might look the same to sonar sensing. Hence, the loop closure search could become less dependent on pose estimates and on sonar inputs, increasing the robustness of SLAM techniques just by utilizing already available data.

Our contribution in this paper is to test if the proposed the use of payload biochemical sensor data for loop closure detection on AUV navigation provides persistent spatiotemporal information that can be used as landmarks.

\section{RELATED WORK}

The body of work concerned with recognizing a place based only on appearance observations within a SLAM framework is known as appearance-based navigation. Two of the most notable examples of such systems are RatSLAM [8] and FAB-MAP 2.0 [9]. These two approaches have proved able to successfully perform appearance-based loop closure detection in large environments of several kilometers. However, they both rely to some extent on a motion 
model of the vehicle, encoded either explicitly as a location prior or implicitly within a biologically inspired navigation model. Attempts to overcome this and perform loop closure detection fully independently from the vehicle DR estimate have been proposed for indoor robots. [10] presented an appearance-based navigation system aimed to detect loop closures without utilizing the robot pose estimate. In [11], Ho et al. derived location descriptors combining both visual and spatial features extracted from synchronized camera images and laser scans collected over a robot path. These descriptors were then used as unique signatures whose similarity among each other was compared through similarity matrices. This similarity and the signatures temporal relationships were then exploited to prompt sequences of loop closures. We share with this work the methodology applied to demonstrate the usability of the spatio-temporal information contained in the water analysis sensors.

Our work aims to apply information from unconventional payload sensors to aid the localization of an autonomous mobile platform. The motivation for this is twofold: i) these sensors might contain useful information to decrease perceptual aliasing and ii) utilizing already available information adds no cost to the system. This is not an original idea and some examples of similar concepts can be found in the field of ground robotics. In [12], Marques et al. explored the use of olfactory sensing to steer a mobile robot towards specific sources of odor. The authors in [13] presented an active localization framework for a mobile platform whose sensing was entirely based on a contact sensor. In a more general approach, [3] tried to determine the minimum set of sensing skills a robot needs to solve the localization problem. The aim of the authors opposed the classical approach to add more and more complex sensory input for localizing, advocating instead for a minimalist approach. This philosophy has motivated the work presented in this paper.

In the underwater domain, Murad Reis et al. introduced in [14] the use of measurements of dissolved oxygen concentration and water turbidity to improve an AUV location estimate in the absence of GPS signal. This is the most closely related work to the one presented here in the sense that it brings into the autonomous localization problem information from more unconventional payload sensors. However, several aspects of their system differ from the one presented here. Firstly, their localization algorithm relies in prior maps of water turbidity, dissolved oxygen and bathymetry of the trajectory. Secondly, their so-called observation points contain depth measurements, which they compare against the prior bathymetry of the AUV trajectory to select re-localization candidates. As they show in the covariance matrix of their dataset, the depth is the variable containing the most descriptive information within their defined observations. This yields their approach very similar to localization based on sparse depth measurements, which [15] proved successful with the use of a Doppler Velocity Log (DVL). In the present work, the descriptors of the areas used to search for loop closure candidates are entirely based on biochemical values and no prior distribution of these are required. Furthermore, we do not propose the use of these measurements to refine the location estimate of the robot, since we do not believe they can provide the require accuracy. Instead, the suggested goal is to utilize them to mitigate perceptual aliasing and increase the robustness of loop closure detection algorithms.

\section{Methodology}

\section{A. Problem definition}

We have an underwater vehicle with a DR system and a suite of $N$ biochemical sensors with measurements at each time interval of $\left\{s_{1}, s_{2}, \ldots, s_{N}\right\}$. In a Bayesian framework, the position estimate at time $t$, of a vehicle with motion model $p_{t}=f\left(p_{t-1}, u_{t}\right)$, and a Gaussian prior can be approximated as a normal distribution given by

$$
p_{t} \sim N\left(f\left(p_{t-1}, u_{t}\right), \Sigma_{t}\right)
$$

Where $p_{t}=[x, y, z]^{T}$ is the position estimate, $u_{t}$ the control input and $\Sigma_{t}$ is the covariance of the distribution. As the vehicle executes the mission, the uncertainty in the position estimate, encoded by $\Sigma_{t}$, increases without bound. Revisiting a previously traversed area where this uncertainty was smaller provides a loop closure constraint, which can be used to correct $p_{t}$ while reducing $\Sigma_{t}$ ([16]). However, in long range missions through unstructured underwater environments, recognizing a revisited area reliably remains a challenge [17].

\section{B. Derivation of the landmarks}

As the vehicle traverses the planned trajectory, payload sensors collect measurements $z_{t}$ of some biochemical processes in the water. These measurements are used to create geo-referenced landmarks $m_{i}$ which consists of a location coordinate and a signature. For all the biochemical sensors, we assume the linear measurement model in Equation 2, since all the sensors analyze the water surrounding the AUV.

$$
m_{i}=\left(\begin{array}{c}
m_{x} \\
m_{y} \\
m_{z} \\
s_{1} \\
\vdots \\
s_{n}
\end{array}\right)=I_{n+3}\left(\begin{array}{c}
p_{x} \\
p_{y} \\
p_{z} \\
s_{1} \\
\vdots \\
s_{n}
\end{array}\right)
$$

With $I$ being the identity matrix of size $n+3$, where $n \in[0, N]$ denotes the number of sensors used to create the signature of the landmark. The index $i=[0, \ldots, T]$ represents the order at which samples where collected throughout the duration $T$ of the mission. We assume here no dynamic model for these sensor readings.

Ideally, when comparing the signatures of the landmarks, high similarity indicates that their location coordinate is the same and hence an area is being revisited. However, for this to work, the signatures must be distinctive enough over space while consistent over time. In order to maximize this uniqueness, thus reducing perceptual aliasing, information 


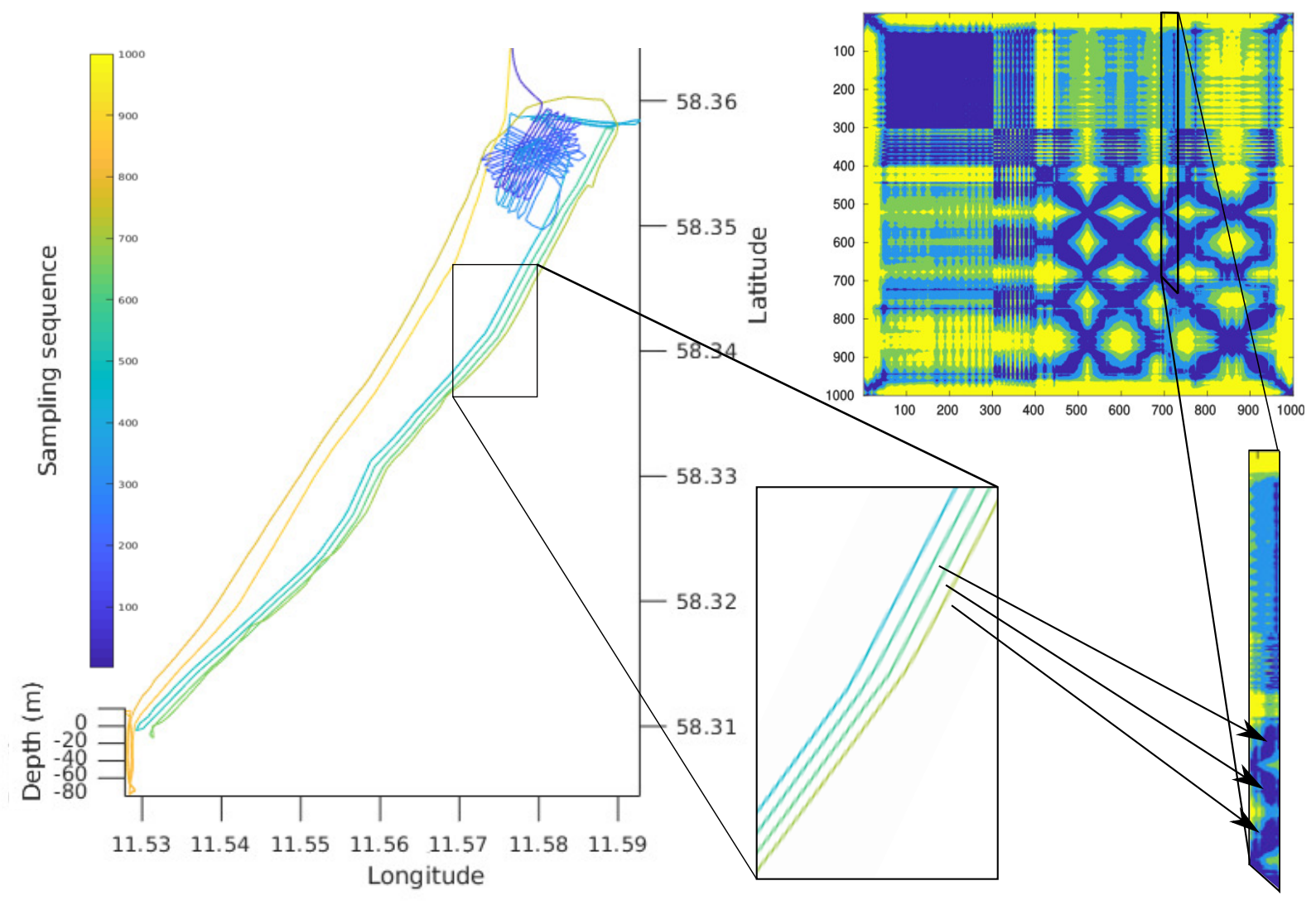

Fig. 1: AUV trajectory for one deployment at Gullmarn fjord and zoom-in of how it relates to its GT similarity matrix.

from other sensors can be added to the descriptor as new dimensions, increasing $n$.

The final size $n$ of the signatures and the sensors selected to conform them in the AUV missions will be determined based on their variances, compared through a principal component analysis (PCA) of all the available sensors. Prior being added to the signature, the sensors are filtered for outliers and interpolated to the 'measurement' time.

\section{Similarity among signatures}

As mentioned above, similarity between landmarks relates to the probability of being at the same place. Given that $S \in$ $\mathbb{R}^{n}$ defines an $n$ dimensional signature space, we can measure similarity as the Mahalanobis distance between signatures in this space as follows

$$
D_{M}\left(S_{i}, S_{j}\right)=\sqrt{\left(S_{i}-S_{j}\right)^{T} \Lambda^{-1}\left(S_{i}-S_{j}\right)}
$$

Where $\Lambda$ is the sample covariance of the signatures distribution. Similarity matrices are a convenient tool to visually depict the results of a comparison of every signature $S_{i}$ against each other for the full AUV trajectory $i=[0, \ldots, T]$. In our case, the aim is to use them to propose loop closures candidates, in a similar fashion to [11].

\section{Information within signatures}

Our hypothesis is that if the biochemical sensors indeed contain information usable for localization, the similarity matrix of the signatures must encode the loop closures occurring along the actual AUV trajectory. In order to compare our approach to the real robot trajectory, ground truth (GT) similarity matrices have been constructed for each mission from the sequences of position estimates $p_{i}$ obtained from the DR system. This has been done since the true positioning of the AUV was not available during all the missions. This is a reasonable assumption given the fact that the particular AUV (see IV-A) used during the missions actually does provide an accurate DR estimate over these missions.

The ground truth similarity matrix from one of the missions presented in Section V can be seen in Figure 1. The color scale of the AUV trajectory encodes the sampling sequence and the similarity matrix has been equalized for visualization purposes. The color scale of the similarity matrix ranges from dark blue to yellow indicating higher to lower similarity respectively. An example of loop closure pattern and how it relates to the vehicle trajectory can be seen in the zoom-in sections of the figure. By the time the AUV is covering a track parallel to the three previous ones, the similarity matrix depicts three dark blue stripes representing three possible loop closures.

In order to quantify how much spatial information is contained in the derived similarity matrices, one must look at the sequences of high similarity values they encode. These can be seen as the structures presented in the matrix when viewed as an image. Hence, the similarity among 
the structures presented in the GT and the derived matrices should give a measure of how much spatial information is contained within the biochemical landmarks. [18] presented a metric used extensively in image quality assessment, the structural similarity index (SSIM), that suits this need. The SSIM index is a combination of local comparison of illumination, contrast and structure across two images divided in equivalent squared windows $x$ and $y$. This combination of the measures takes the form

$$
\operatorname{SSIM}(x, y)=[l(x, y)]^{\alpha} \cdot[c(x, y)]^{\beta} \cdot[s(x, y)]^{\gamma}
$$

Where $l(x, y)$ represents the luminance, $c(x, y)$ the contrast and $s(x, y)$ is the structure measure, and the parameters $\alpha>0$, $\beta>0$ and $\gamma>0$ are used to adjust their weights. The standard values for these weight are $\alpha=\beta=\gamma=1$. However, since we only care about the underlying structure encoded in the images, $\alpha$ and $\beta$ have been set to zero when comparing similarity matrices.

\section{FIELD TESTS}

This section describes the biochemical sensors studied, the AUV platform used and the missions carried out to collect the datasets.

\section{A. AUV platform and sensor payload}

The water analysis sensors together with their output used for the experiments are listed below

- CTD: Conductivity and temperature

- ECO FLBBCD: Backscatter, ChlA and FDOM

- CONTROS HydroC CO2: dissolved $\mathrm{CO}_{2}$

- Deep SUNA: dissolved $\mathrm{NO}_{3}$

These sensors were set as passive payload and so the data collection did not alter the course of the mission.

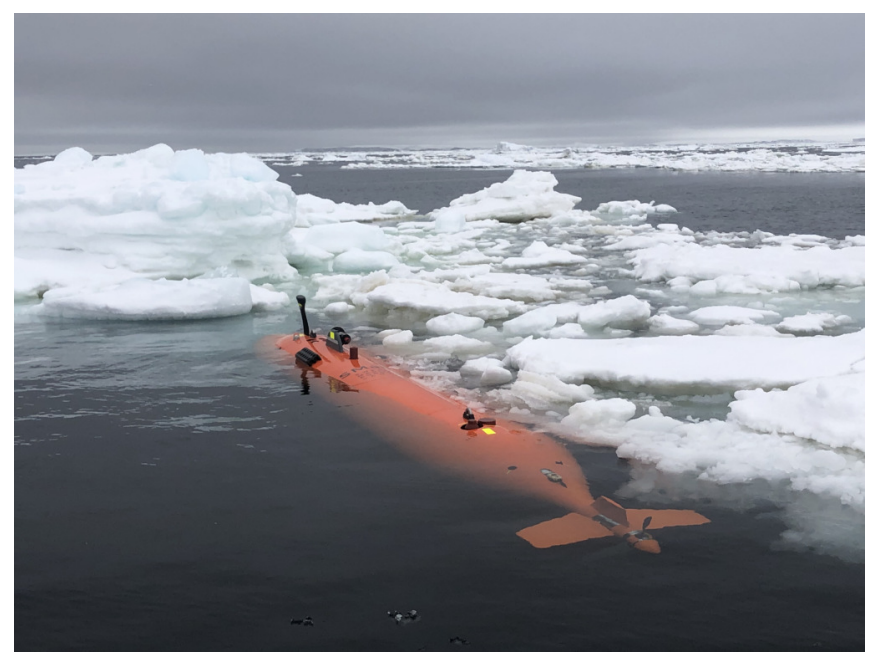

Fig. 2: Hugin AUV deployed at Thwaites Glacier ${ }^{1}$.

The missions were carried out by an AUV Kongsberg Hugin (Fig. 2) operated in autonomous mode. During the missions in the Gullmarn fjord no acoustic positioning or

\footnotetext{
${ }^{1}$ Photography courtesy of Filip Stedt.
}

terrain-aided navigation system were available. This means that the position estimate $p_{t}$ was entirely provided by the inertial navigation system (INS) of the vehicle, which fuses IMU and DVL measurements through an extended Kalman filter. For the missions in Thwaites glacier an acoustic beacon Kongsberg cNode Maxi was employed to aid the localization.

\section{B. Locations and missions}

The data collections have taken place in two different geographic locations to generalize results more reliably.

1) The Gullmarn Fjord: The first scenario was the Gullmarn Fjord, on the west coast of Sweden, during the autumn of 2018. This area is a good testbed for this kind of experiments since the behavior and distribution of the biochemical markers under study is well understood [19], [20]. ChlA, backscatter and FDOM are highly season dependent. Most of the markers used, i.e. salinity, temperature, ChlA, backscatter or FDOM, have their greatest variation in the vertical in the Gullmarn Fjord and can be regarded as stationary for each water mass during the missions.

2) The Thwaites glacier: The Thwaites glacier, in the Amundsen sea, is an Antarctic glacier whose stability has been compromised in recent years. As a part of an expedition on board the Nathaniel B. Palmer icebreaker in January 2019 , a Hugin AUV has been deployed in several missions beneath the glacier front to collect biological data in such a remote and unstudied area. These payload data has been analyzed in this work in order to find out if it can potentially be utilized for the autonomous localization of the AUV in unexplored and unknown environment such as underneath a glacier.

TABLE I: Missions

\begin{tabular}{|c|c|c|c|}
\hline Mission & Location & Duration $(\mathrm{h})$ & Payload Sensors \\
\hline 1 & Gullmarn & 4.7 & CTD, FLBBCD, NO3 \\
2 & Thwaites & 17.5 & CTD, FLBBCD, NO3, CO2 \\
3 & Gullmarn & 19.1 & CTD, FLBBCD, NO3 \\
4 & Gullmarn & 6.7 & CTD, FLBBCD, NO3 \\
\hline
\end{tabular}

\section{EXPERIMENTAL RESULTS}

This section presents the results on the data collected in the campaigns in Table I.

\section{A. Landmarks signature}

According to the PCA analyses on the amount of information contained in the sensor data, only the FLBBCD has proved useful for localization according to the criteria established in Section III. Thus, the results presented hereafter have all been obtained with landmarks whose signature was of size $n=3$ and was constructed combining only fluorescence, FDOM and scattering measurements.

\section{B. Resulting similarity matrices}

The similarity matrices generated from the missions in Table I and the trajectories followed by the AUV while collecting the data can be seen in Figures 6-9. The color code of the AUV trajectories and the matrices follows the format 
explained in Section III-D. The missions presented have been selected based on the complexity of the AUV trajectory in terms of steering, length and area covered. All of them have a duration long enough to help determine if the markers are consistent over long-range mission. Mission 1 and 2 have a wider depth range than 3 and 4, for which the AUV was set to keep a constant altitude and hence contain less variation in the depth scale. This was done to help determine the dependency of the biochemical markers with the depth and how it influences the usability of the landmarks. Mission 3 is the longest one and describes an area coverage pattern of parallel lines over a large area. In this case, there are not actual loop closures in the trajectory since the swaths are parallel, but they are close enough to each other as to share similar values of the biochemical markers. Mission 4 is probably the most challenging one since it covers repeatedly a very confined area.

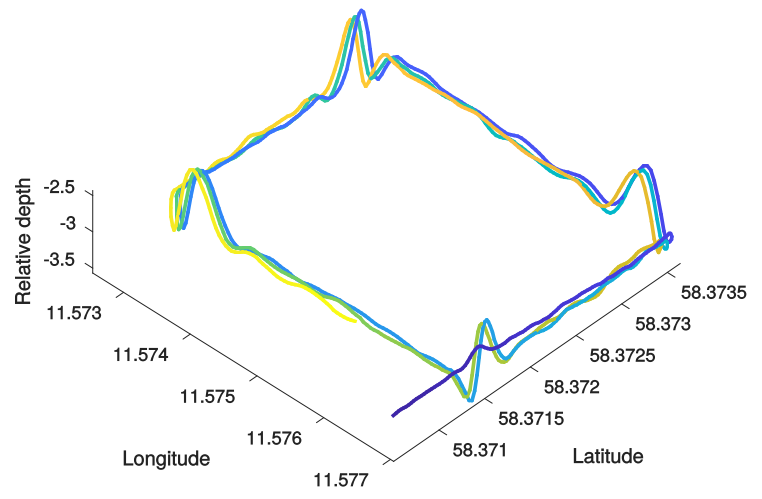

Fig. 3: A zoomed in section of mission 1 with 3 loops.

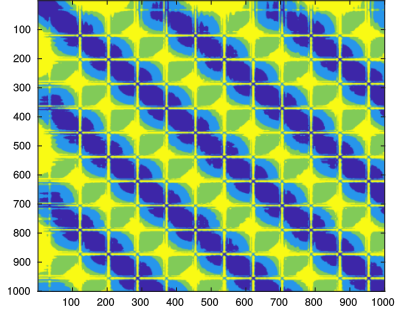

(a) Ground truth

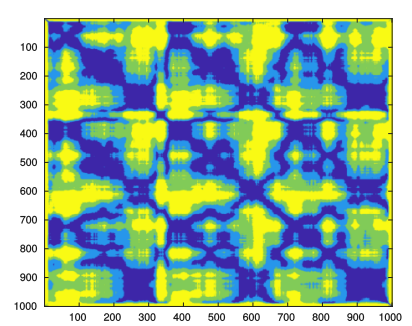

(b) Biochemical signatures
Fig. 4: Similarity matrices corresponding to Fig 3

\section{Spatial resolution of the landmarks}

In order to quantify the minimum distance among landmarks at which they could provide a distinctive enough signature, a segment of the AUV trajectory in mission 1 in Fig. 6 is analyzed. The AUV track, depicted in Fig. 3, consists of three overlapping loops at constant depth in an area of $260 \times 450$ meters. The two continuous loop closure sequences starting approximately at sample $i=350$ and $i=650$ can be easily seen in the ground truth similarity matrix in 4 . The same patterns arise in the raw similarity matrix composed of the biochemical signatures, although distorted. This indicates the presence of usable information in the defined landmarks and thus, that they could be utilized to detect loop closure sequences even in such confined areas.

\section{Comparison with ground truth}

The SSIM index has been used to measure how well the biochemical landmarks can be used to detect the loop closure sequences present in the ground truth data. The results can be seen in Table II together with the number of samples.

TABLE II: Missions

\begin{tabular}{|c|c|c|}
\hline Mission & Samples $(i)$ & SSIM \\
\hline 1 & 9000 & 0.855 \\
2 & 8000 & 0.832 \\
3 & 9000 & 0.719 \\
4 & 8000 & 0.550 \\
\hline
\end{tabular}

With $\operatorname{SSIM} \in[0,1]$, where 1 denotes full equality of the images compared. The local SSIM indexes resulting from the comparisons are depicted in Figure 5, with the brightness indicating the magnitude of the index. Intuitively, the whiter the image, the higher the structure similarity and hence the more structural information the biochemical landmarks preserve. A close look at Figure 5 shows that the ground truth sequences of loop closures have been preserved within the derived similarity matrices, with the worst performance corresponding to mission 4 , as expected.

\section{CONCLUSIONS AND FURTHER WORK}

This work has introduced the preliminary results from a study of the potential and viability of utilizing payload biochemical sensors to contribute loop closure candidates to an AUV localization framework. A method to build and compare biochemical landmarks has been presented and several water analysis sensors have been examined across missions in two different geographical areas. These measurements are highly season-dependent and are not aimed to be used as static maps across missions. Instead, the aim is to provide a more robust data association across a single mission.

Initial results seem to indicate that the FLBBCD is the only sensor among the ones tested containing usable information for all our tests. An analysis of the SSIM structural preservation of the loop closure sequences in similarity matrices generated from these sensor seems to indicate they could indeed be used in a loop closure detection framework for underwater SLAM. Potential spatial resolution in areas as small as $260 \times 450$ meters has been proved.

Further work will focus on proving if these results can be generalized by testing the approach with data collected in a broader range of environments. Furthermore, this method will be implemented in a fully probabilistic SLAM framework to show how it can be incorporated to an AUV system.

\section{ACKNOWLEDGMENT}

This work was supported by Stiftelsen for Strategisk Forskning (SSF) through the Swedish Maritime Robotics 
Centre (SMaRC) (IRC15-0046). We would also like to acknowledge the Knut and Alice Wallenberg foundation for funding MUST, Mobile Underwater System Tools, project that provided the Hugin AUV for these tests.

\section{REFERENCES}

[1] A. W. Stoner, C. H. Ryer, S. J. Parker, P. J. Auster, and W. W. Wakefield, "Evaluating the role of fish behavior in surveys conducted with underwater vehicles," Canadian Journal of Fisheries and Aquatic Sciences, vol. 65, no. 6, pp. 1230-1243, 2008.

[2] M. Solan, J. D. Germano, D. C. Rhoads, C. Smith, E. Michaud, D. Parry, F. Wenzhöfer, B. Kennedy, C. Henriques, E. Battle, et al., "Towards a greater understanding of pattern, scale and process in marine benthic systems: a picture is worth a thousand worms," Journal of experimental marine biology and ecology, vol. 285, pp. 313-338, 2003.

[3] J. M. O'Kane and S. M. LaValle, "Localization with limited sensing," IEEE Transactions on Robotics, vol. 23, no. 4, pp. 704-716, 2007.

[4] C. Roman and H. Singh, "Improved vehicle based multibeam bathymetry using sub-maps and slam," in Intelligent Robots and Systems, 2005.(IROS 2005). 2005 IEEE/RSJ International Conference on, pp. 3662-3669, IEEE, 2005.

[5] C. Beall, F. Dellaert, I. Mahon, and S. B. Williams, "Bundle adjustment in large-scale $3 \mathrm{~d}$ reconstructions based on underwater robotic surveys," in OCEANS, 2011 IEEE-Spain, pp. 1-6, IEEE, 2011.

[6] J. J. Leonard and A. Bahr, "Autonomous underwater vehicle navigation," in Springer Handbook of Ocean Engineering, pp. 341-358, Springer, 2016.

[7] N. Gruber and S. Doney, "Modeling of ocean biogeochemistry and ecology," 2018.

[8] M. Milford and G. Wyeth, "Mapping a suburb with a single camera using a biologically inspired slam system," IEEE Transactions on Robotics, vol. 24, no. 5, pp. 1038-1053, 2008.

[9] M. Cummins, "Highly scalable appearance-only slam-fab-map 2.0," Proc. Robotics: Sciences and Systems (RSS), 2009, 2009.

[10] M. Cummins and P. Newman, "Probabilistic appearance based navigation and loop closing," in Proceedings 2007 IEEE International Conference on Robotics and Automation, pp. 2042-2048, IEEE, 2007.

[11] K. L. Ho and P. Newman, "Loop closure detection in slam by combining visual and spatial appearance," Robotics and Autonomous Systems, vol. 54, no. 9, pp. 740-749, 2006.

[12] L. Marques, U. Nunes, and A. T. de Almeida, "Olfaction-based mobile robot navigation," Thin solid films, vol. 418, no. 1, pp. 51-58, 2002.

[13] L. H. Erickson, J. Knuth, J. M. O'Kane, and S. M. LaValle, "Probabilistic localization with a blind robot," in 2008 IEEE International Conference on Robotics and Automation, pp. 1821-1827, IEEE, 2008.

[14] G. M. Reis, H. Leon, T. Alam, J. Anderson, L. Bobadilla, and R. N. Smith, "A whitening-based tracking algorithm for autonomous underwater vehicles," in 2018 OCEANS-MTS/IEEE Kobe TechnoOceans (OTO), pp. 1-6, IEEE, 2018.

[15] V. Bichucher, J. M. Walls, P. Ozog, K. A. Skinner, and R. M. Eustice, "Bathymetric factor graph slam with sparse point cloud alignment," in OCEANS 2015-MTS/IEEE Washington, pp. 1-7, IEEE, 2015.

[16] M. F. Fallon, J. Folkesson, H. McClelland, and J. J. Leonard, "Relocating underwater features autonomously using sonar-based slam," IEEE Journal of Oceanic Engineering, vol. 38, no. 3, pp. 500-513, 2013.

[17] P. L. Negre, F. Bonin-Font, and G. Oliver, "Cluster-based loop closing detection for underwater slam in feature-poor regions," in 2016 IEEE International Conference on Robotics and Automation (ICRA), pp. 2589-2595, IEEE, 2016.

[18] Z. Wang, A. C. Bovik, H. R. Sheikh, E. P. Simoncelli, et al., "Image quality assessment: from error visibility to structural similarity," IEEE transactions on image processing, vol. 13, no. 4, pp. 600-612, 2004.

[19] R. Parsmar and A. Stigebrandt, "Observed damping of barotropic seiches through baroclinic wave drag in the gullmar fjord," Journal of Physical Oceanography, vol. 27, no. 6, pp. 849-857, 1997.

[20] H. L. Filipsson and K. Nordberg, "Climate variations, an overlooked factor influencing the recent marine environment. an example from gullmar fjord, sweden, illustrated by benthic foraminifera and hydrographic data," Estuaries, vol. 27, no. 5, pp. 867-881, 2004.

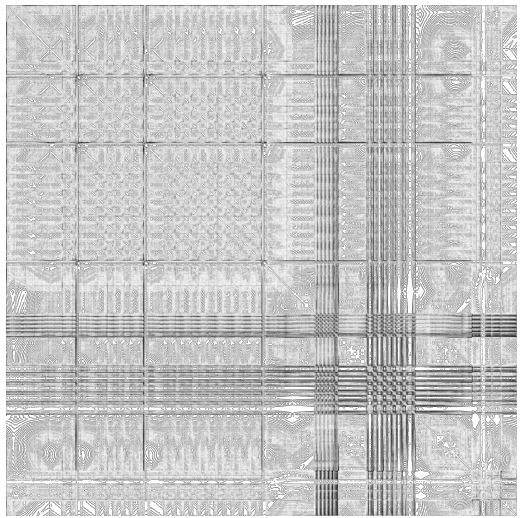

(a) SSMI result for mission 1, in Fig. 6

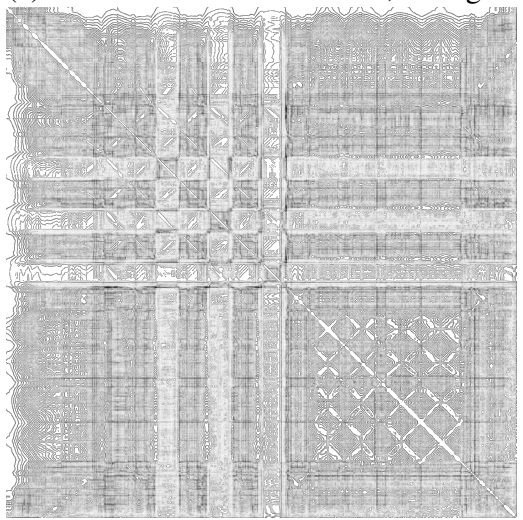

(b) SSMI result for mission 2, in Fig. 7

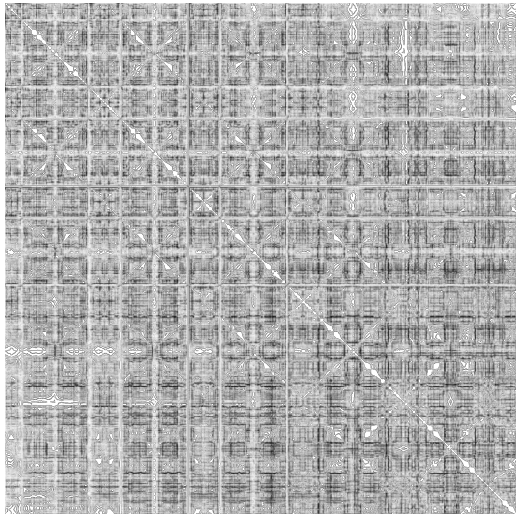

(c) SSMI result for mission 3, in Fig. 8

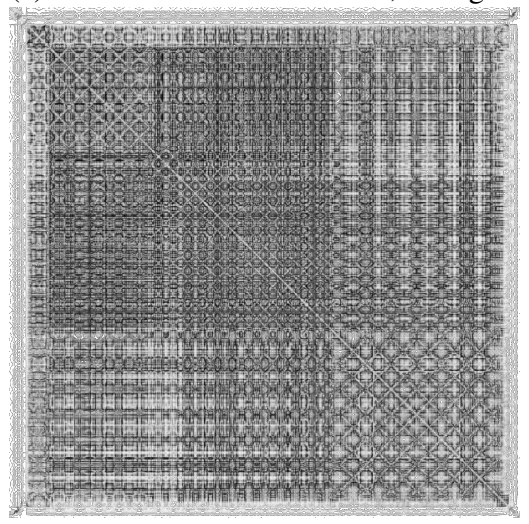

(d) SSMI result for mission 4, in Fig. 9

Fig. 5: SSIM comparison of GT and derived similarity matrices in Figures 6-9. 


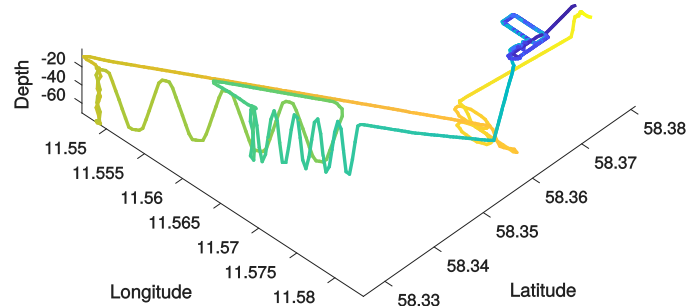

(a) AUV trajectory

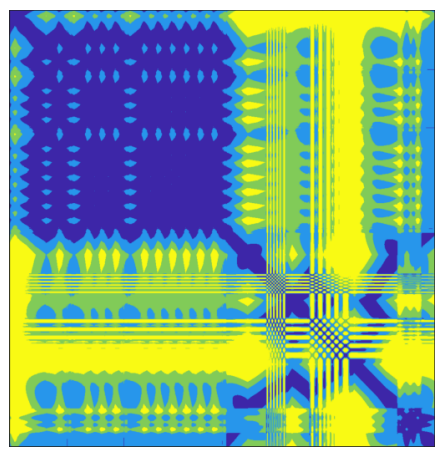

(b) Ground truth

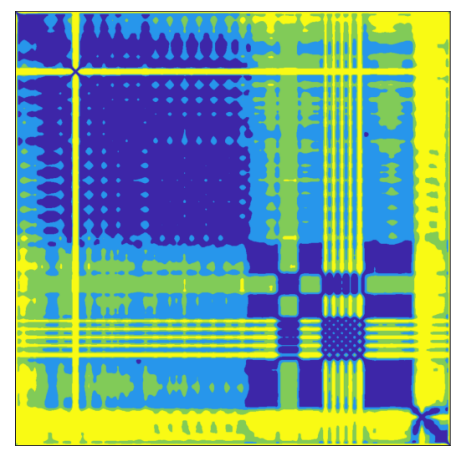

(c) Biochemical signatures

Fig. 6: Trajectory and similarity matrices from mission 1

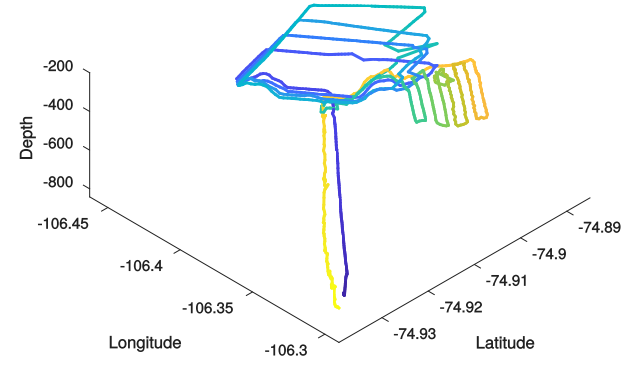

(a) AUV trajectory

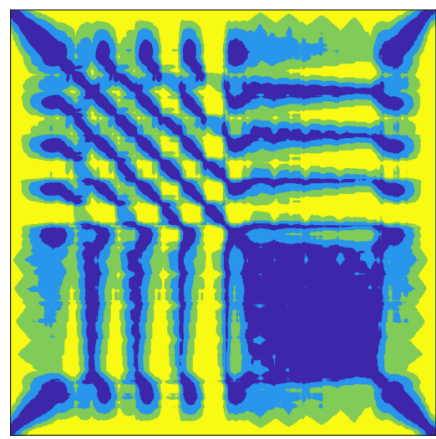

(b) Ground truth

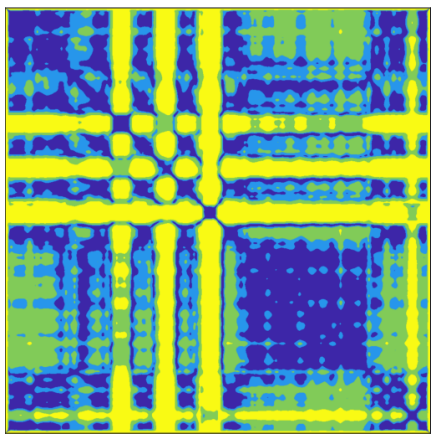

(c) Biochemical signatures

Fig. 7: Trajectory and similarity matrices from mission 2

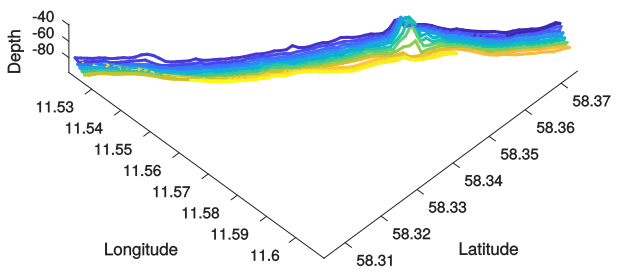

(a) AUV trajectory

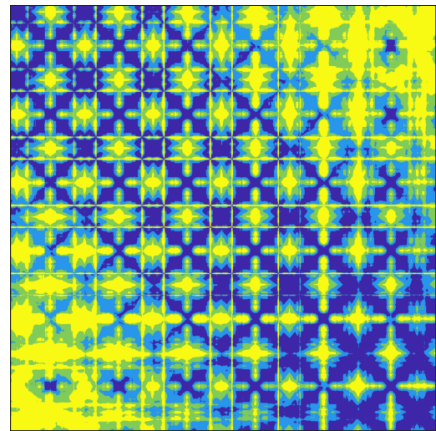

(b) Ground truth

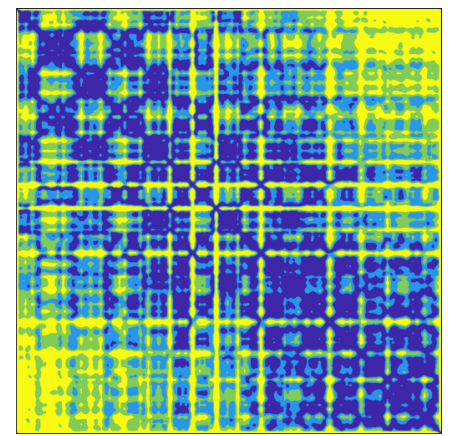

(c) Biochemical signatures

Fig. 8: Trajectory and similarity matrices from mission 3

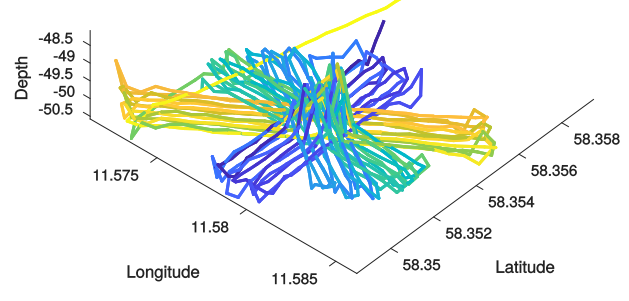

(a) AUV trajectory

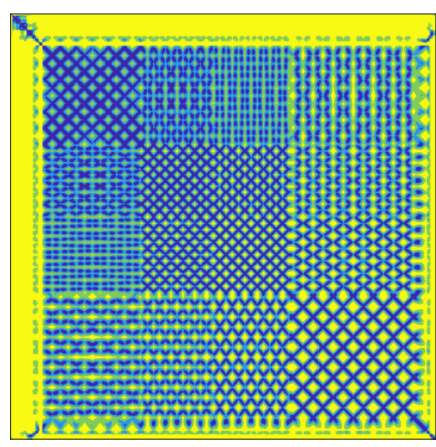

(b) Ground truth

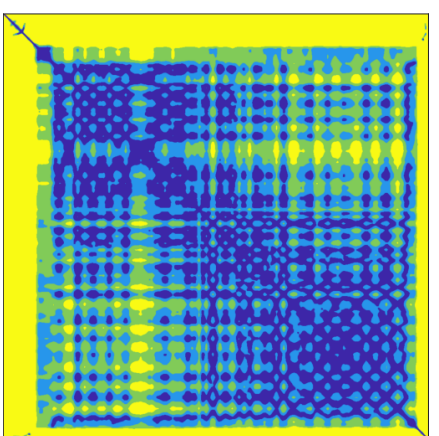

(c) Biochemical signatures

Fig. 9: Trajectory and similarity matrices from mission 4 